\title{
A Reference Sample: ISM of the Most Isolated Galaxies
}

\author{
L. Verdes-Montenegro
}

Instituto de Astrofísica de Andalucía-CSIC, Granada, Spain

J. Sulentic

Physics \& Astronomy, University of Alabama, USA

D. Espada, S. Leon, U. Lisenfeld, S. Verley

Instituto de Astrofísica de Andalucía-CSIC, Granada, Spain

W. Huchtmeier

Max-Planck-Institut für Radioastronomie, Bonn, Germany

S. Odewahn

Department of Physics and Astronomy, Arizona State University, USA

E. Garcia

Instituto de Astrofísica de Andalucía-CSIC, Granada, Spain

M. S. Yun

Department of Physics and Astronomy, University of Massachusetts, USA

S. del Río

INAOE, Puebla, Mexico

F. Combes

Observatoire de Paris - LERMA, France

\begin{abstract}
We are constructing the first complete unbiased control sample of the most isolated galaxies of the northern sky to serve as a template in the study of star formation and galaxy evolution in denser environments. Our goal is to compare and quantify the properties of different phases of the interstellar medium in this sample, as well as the level of star formation, both relevant parameters in the internal evolution of galaxies and strongly conditioned by the environment. To achieve this goal we are building a multiwavelength database for this sample to compare and quantify the properties of different phases of the ISM.
\end{abstract}




\section{The CIG refined sample}

Our source list is drawn from the Catalogue of Isolated Galaxies (CIG; Karachentseva 1973) and our sample contains 800 galaxies (Verdes-Montenegro et al 2001, 2002). Advantages of our CIG-based sample include isolation, morphological diversity, depth and completeness (reasonably complete out to $\mathrm{V}_{0}=10000 \mathrm{~km} / \mathrm{s}$ ). We cover a range of optical luminosities of $\log \left(\mathrm{L}_{B} / \mathrm{L}_{\text {sol }}\right)=8-11.2$. We are currently refinining positions, velocities and isolation definition of the sample. We revised positions for the $1051 \mathrm{CIG}$ galaxies. Original measurements come from different sources and we have calculated the new positions in a uniform way. We have found differences between the older and newer coordinates of up to 44.5 arcsec with a mean value of 1.8 arcsec (Leon \& Verdes-Montenegro 2003). We obtained the best available redshifts for 926 CIGs from different surveys in the bibliography increasing by 458 those used in previous CIG based papers. We are using the publicly available images of POSS-I E plates (as well as POSS-II F plates for some fields) in order to revise the isolation degree of CIG galaxies.

\section{Multiwavelength characterization of the ISM}

Our database will emphasize the properties of the nonstellar material in the galaxies because it is most sensitive to the effects of external stimuli. The chosen parameters are: Optical luminosity as tracer of the stellar content, molecular gas, from which stars form, $\mathrm{H}_{\alpha}$, a good tracer of stellar formation where extinction is not high, atomic gas, as a very sensitive component in interacting galaxies, radio-continuum emission as a very useful free-from-extinction tool to determine current SFR in a spiral galaxy and possible nuclear activity, and far-infrared emission, where most of the flux from forming stars is re-radiated. We plan to extend our wavelength range to the NIR in the near future. It is not realistic to obtain $\mathrm{CO}$ and $\mathrm{HI}$ data for the whole sample and for their study we will focus on a smaller redshift-limited subsample $(1500<\mathrm{v}<5000 \mathrm{~km} / \mathrm{s})$ composed of 206 galaxies, much preferable from a flux-limited sample which unavoidably leads to biases.

A Web interface is being prepared to make the survey database available to the community.

Acknowledgments. This work is partially supported by DGI Grant AYA 2002-03338.

\section{References}

Karachentseva, I. 1973, Comm. Spec. Ap. Obs., USSR, 8, 1

Leon, S. \& Verdes-Montenegro, L. 2003, A\&A, 411, 391

Verdes-Montenegro, L., Sempere, M. J., Sulentic, J., \& Cernicharo, J. 2001, ESASP, 460, 515

Verdes-Montenegro, L., Sauvage, M., Sempere, M. J., Sulentic, J., \& Cernicharo, J., 2002, AP\&SS, 281, 1, 427 